\title{
The attributable mortality of delirium in critically ill patients: prospective cohort study
}

\author{
(c) (1) (8) OPEN ACCESS
}

\author{
Peter M C Klein Klouwenberg PhD student ${ }^{1}$, Irene J Zaal PhD student ${ }^{1}$, Cristian Spitoni statistician ${ }^{2}$, \\ David S Y Ong PhD student ${ }^{1}$, Arendina W van der Kooi clinical technologist ${ }^{1}$, Marc J M Bonten \\ epidemiologist ${ }^{3}$, Arjen J C Slooter neurologist-intensivist ${ }^{1}$, Olaf L Cremer anaesthesiologist-intensivist ${ }^{1}$
}

'Department of Intensive Care Medicine, University Medical Centre Utrecht, 3508 GA, Utrecht, Netherlands; ${ }^{2}$ Department of Mathematics, Utrecht University, Utrecht, Netherlands; ${ }^{3}$ Department of Medical Microbiology, University Medical Centre Utrecht, Utrecht, Netherlands

\begin{abstract}
Objective To determine the attributable mortality caused by delirium in critically ill patients.

Design Prospective cohort study.

Setting 32 mixed bed intensive care unit in the Netherlands, January 2011 to July 2013.
\end{abstract}

Participants 1112 consecutive adults admitted to an intensive care unit for a minimum of 24 hours.

Exposures Trained observers evaluated delirium daily using a validated protocol. Logistic regression and competing risks survival analyses were used to adjust for baseline variables and a marginal structural model analysis to adjust for confounding by evolution of disease severity before the onset of delirium.

Main outcome measure Mortality during admission to an intensive care unit.

Results Among 1112 evaluated patients, 558 (50.2\%) developed at least one episode of delirium, with a median duration of 3 days (interquartile range 2-7 days). Crude mortality was 94/558 (17\%) in patients with delirium compared with $40 / 554(7 \%)$ in patients without delirium $(P<0.001)$. Delirium was significantly associated with mortality in the multivariable logistic regression analysis (odds ratio 1.77, 95\% confidence interval 1.15 to 2.72 ) and survival analysis (subdistribution hazard ratio $2.08,95 \%$ confidence interval 1.40 to 3.09 ). However, the association disappeared after adjustment for time varying confounders in the marginal structural model (subdistribution hazard ratio 1.19, 95\% confidence interval 0.75 to 1.89$)$. Using this approach, only $7.2 \%(95 \%$ confidence interval $-7.5 \%$ to $19.5 \%$ ) of deaths in the intensive care unit were attributable to delirium, with an absolute mortality excess in patients with delirium of $0.9 \%$ ( $95 \%$ confidence interval $-0.9 \%$ to $2.3 \%$ ) by day 30. In post hoc analyses, however, delirium that persisted for two days or more remained associated with a $2.0 \%$ (95\% confidence interval $1.2 \%$ to $2.8 \%$ ) absolute mortality increase. Furthermore, competing risk analysis showed that delirium of any duration was associated with a significantly reduced rate of discharge from the intensive care unit (cause specific hazard ratio $0.65,95 \%$ confidence interval 0.55 to 0.76 ).

Conclusions Overall, delirium prolongs admission in the intensive care unit but does not cause death in critically ill patients. Future studies should focus on episodes of persistent delirium and its long term sequelae rather than on acute mortality.

Trial registration Clinicaltrials.gov NCT01905033.

\section{Introduction}

Delirium is a common complication of critical illness, occurring in $30-60 \%$ of patients admitted to an intensive care unit. ${ }^{1-5}$ Although most studies have identified delirium as an independent predictor of death in the intensive care unit, ${ }^{6-14}$ several others found no association with mortality. ${ }^{15-17}$ These inconsistencies have been explained by differences in case mix, the tools used for the assessment of delirium, and the study design. ${ }^{18}$ Deficiencies in modelling methodology and residual confounding may, however, provide an alternative explanation. In particular, none of the previous studies have adequately adjusted for disease progression before the start of delirium, or for competing events (such as discharge) that may preclude observation of mortality in the intensive care unit. It therefore remains unclear whether delirium is merely a marker of poor prognosis or causally linked to mortality in the intensive care unit.

We estimated the proportion of deaths that can be attributed to delirium in a large cohort of critically ill patients by performing a marginal structural model analysis from the discipline of causal inference. Such analysis can overcome bias that results from the evolution of disease severity until the onset of delirium as 
well as more traditional sources of bias. ${ }^{19}{ }^{20}$ To aid in the interpretation of our findings, we compared the results of the marginal structural model analysis with those of standard statistical regression methods.

\section{Methods \\ Study population}

We prospectively evaluated consecutive adults admitted for at least 24 hours to the 32 mixed bed intensive care unit of the University Medical Centre Utrecht, the Netherlands, between January 2011 and June 2013. We excluded patients with acute or premorbid neurological disease at baseline, those in whom assessments of delirium could not be performed owing to a language barrier, and those transferred from or to another intensive care unit. The local ethical review board gave approval for an opt-out consent method (institutional review board number 10-056/12-421) whereby participants and family members were notified of the study by a brochure that was provided at admission to the intensive care unit with an attached opt-out card.

\section{Delirium}

A research team dedicated to this study used a validated flowchart to classify the mental status of patients daily until discharge from intensive care. ${ }^{21}$ All relevant information was available to the study team, including the 12 hourly confusion assessment method for the intensive care unit conducted by nurses. We categorised patients as comatose, sedated, awake and delirious, or awake and non-delirious. Firstly, we assessed the level of consciousness using the Richmond agitation-sedation scale. Patients with maximum scores of -5 or -4 during the entire 24 hour observation period could not be assessed for delirium and were classified as either comatose or sedated. ${ }^{22} \mathrm{~A}$ sedated state was defined as propofol continuously administered at a rate of $>1 \mathrm{mg} / \mathrm{kg} / \mathrm{h}$ and/or midazolam at a dose of $>50 \mathrm{mg} / \mathrm{d}$ or equivalent either at the time of assessment or at any time in the 48 hours before assessment. All other patients with scores of -5 or -4 on the Richmond agitation-sedation scale were classified as comatose. We assessed the remaining patients for delirium using the confusion assessment method for the intensive care unit as well as inspection of medical notes and nursing charts by the research team. These patients were classified as delirious when they tested positive on the confusion assessment method in the intensive care unit and/or when there was a description of fluctuation in the level of consciousness, agitation, disorientation, or hallucinations. Furthermore, because haloperidol and quetiapine were exclusively used for the treatment of delirium during the study period, we also classified patients as delirious on the day of initiation of either of these drugs. In case of doubt, a neurologist (AS) was consulted, who cast the decisive vote for classification of mental status. This procedure had a sensitivity of $0.75(95 \%$ confidence interval 0.47 to 0.92 ), specificity of 0.85 (95\% confidence interval 0.68 to 0.94 ), and an excellent inter-rater agreement (Fleiss' $\kappa 0.94$ ) (unpublished data). To enable our primary analysis we dichotomised the mental status by reclassifying sedated patients as non-delirious and comatose patients (without sedation) as delirious (see supplementary figure 1). The clinical team responsible for the patients was unaware of the results of the delirium assessments made by the study team.

\section{Covariables and outcome}

In all multivariable models we adjusted for covariables that we chose a priori based on their expected associations with delirium and mortality after careful consideration of the literature. These covariables included age, sex, history of dementia, history of alcohol misuse, Charlson comorbidity index, acute physiology and chronic health evaluation IV score, admission type, readmission status, and presence of sepsis on admission to the intensive care unit. ${ }^{23}$ These are all time fixed variables, representing the risk of delirium at baseline. However, because the risk of delirium onset is likely to vary over the course of admission to an intensive care unit depending on the evolution of disease severity, we also incorporated time dependent variables in our primary analysis (fig $1 \Downarrow$ ). These included daily measurements of the sequential organ failure assessment score, sepsis status, core temperature, mechanical ventilation status, use of sedative and analgesic drugs, and plasma sodium, urea, acidosis, and haematocrit levels. ${ }^{167151724-38}$ Several physiological and laboratory variables (temperature, sodium, urea, and haemoglobin) were transformed to account for their non-linear relation with delirium or mortality, using the cut-offs from the acute physiology and chronic health evaluation IV model. ${ }^{39}$ Observers dedicated to this study collected data prospectively as part of a large cohort study and regularly checked for data integrity. ${ }^{40}$

No data were missing for baseline variables, daily mental status classifications, or the outcome. However, for daily observations of several laboratory and physiological variables, $3.1 \%$ of data were missing overall (range $0-6.9 \%$ for individual variables). Because of the availability of longitudinal data before each observation day for each patient, we performed a trend imputation for missing covariables. ${ }^{41}$ Mortality during admission to an intensive care unit was the primary outcome of interest in all analyses. Although long term sequelae of delirium are known to exist, in the present study we deliberately focused on short term outcomes because previous authors have claimed up to a threefold increased mortality rate in the intensive care unit even after correction for confounders. ${ }^{18}$ Furthermore, adjustment for time dependent covariables using marginal structural models requires daily information about severity of disease and therapeutic interventions for the duration of observation. Practically, this precludes the use of outcomes that lie beyond discharge from the intensive care unit.

\section{Statistical analysis}

To obtain first estimates of the association between delirium and mortality in our cohort, and to be able to compare our results with previous literature, we performed a multivariable logistic regression analysis, adjusting for a priori selected baseline confounders. To comply with existing literature, we assumed that patients who develop delirium are at increased risk for the duration of their stay in the intensive care unit, even if delirium develops only several days after admission. The resulting bias can be overcome by using a Cox proportional hazards analysis and with inclusion of delirium as a time dependent variable. ${ }^{42}$ In this type of analysis, however, informative censoring of the survival time should additionally be taken into account by considering discharge as a competing risk for mortality, because patients who are discharged from the intensive care unit alive are in a different health state from patients who remain admitted beyond that time point. ${ }^{43} 44 \mathrm{~A}$ competing risks analysis provides two measures of association: the cause specific hazard ratio, which in this case estimates the direct effects of delirium on outcome (both intensive care unit discharge and death), and the subdistribution hazard ratio, which describes the instantaneous risk of dying from delirium given that the patient has not died from delirium. ${ }^{45}$ The subdistribution hazard ratio is therefore a summary measure of all separate cause specific hazards and can 
be used to calculate the cumulative incidence of the outcome of interest (that is, death in this study). Although the methods can adjust for baseline confounders, the time varying nature of delirium onset and informative censoring caused by discharge from the intensive care unit, a limitation of these methods is that neither can adjust for other, potentially important sources of confounding. Firstly, the severity of disease on the day of admission to the intensive care unit may not be representative of the health state at the time of delirium onset, which typically occurs later on during stay in the intensive care unit (fig 1). As delirium preferentially develops in patients who are more severely ill, ${ }^{25}$ bias occurs when such changes in disease severity are not adjusted for during the analysis. Secondly, bias might occur when a time dependent covariable is not only a risk factor for death but also predicts subsequent delirium, and when delirium status at a previous time point predicts the risk factor. ${ }^{46}$ For instance, severely agitated patients with delirium may eventually be treated with sedatives, whereas sedative use itself is a known risk factor for delirium. ${ }^{25}$ A marginal structural model analysis deals with these limitations by adjusting for the changes in disease severity before delirium onset, while preventing bias. ${ }^{19}{ }^{20}$ It enables assessment of what the mortality in the intensive care unit would have been in a hypothetical population in which all patients remained delirium-free, and is therefore called a counterfactual analysis.

To accomplish such a counterfactual analysis, we performed two steps. Firstly, we modelled the daily probability of acquiring delirium in the intensive care unit, using a multivariable logistic regression analysis that included both baseline and daily patient characteristics. Based on these estimated daily probabilities, we calculated stabilised patient specific weights (so called inversed probability weights) that represent the cumulative risk of acquiring delirium for each patient. Because adjustment for time varying variables measured after the start of delirium may result in bias, we used lagged values from the preceding day to predict delirium on each day. ${ }^{47} \mathrm{We}$ adjusted for lagged values of the sequential organ failure assessment scores two days before to acknowledge that the scores measured within 24 hours before the onset of delirium may have been influenced by an insidious onset of delirium. Secondly, we performed an inverse probability weighted Cox regression analysis with competing endpoints (death and discharge alive) and estimated both the daily hazard and cumulative risk of death. To aid in the interpretation of the results, we computed the population attributable fraction, which indicates the percentage of patients who have died from delirium.

We performed several post hoc sensitivity analyses (see supplementary figure 1). Firstly, instead of categorising sedated patients as non-delirious, we reclassified these patients based on the first available valid assessment for delirium after the cessation of sedation, using backward imputation. Secondly, we applied a more rigorous definition of delirium by considering patients as being delirious only when they had been classified as delirious on at least two consecutive days. Thirdly, to assess possible effect modification by the underlying condition, we performed subgroup analyses in patients with sepsis only, and stratified by acute physiology and chronic health evaluation IV score.

All analyses were performed using SAS 9.2 (Cary, NC) and R 2.14 (www.r-project.org). We used the R-package "IPW" 48 for the marginal structural model analysis. $\mathrm{P}$ values less than 0.05 were considered to be statistically significant. We used robust estimators (Huber sandwich) to calculate confidence intervals for the hazard ratios resulting from the marginal structural model analyses,${ }^{49}$ and we used bootstrapping to estimate the confidence intervals for the attributable mortality.

\section{Results}

During the 2.5 year study period, 2854 critically ill patients were admitted to our intensive care unit of whom 1112 met the inclusion criteria (fig $2 \Downarrow$ ). The average length of stay was 8.9 days, amounting to a total of 9867 observation days. Delirium was observed on 2524 (26\%) of these days. However, to obtain a dichotomous exposure variable for use in the regression analyses, we additionally reclassified 537 coma days to days with delirium and 1371 sedation days to days without delirium. After this, 558 (50\%) patients had at least one episode of delirium. The median time to onset of delirium was 2.0 days (interquartile range 1.0-4.0 days) and the median duration of a delirium episode was 3.0 (interquartile range 2.0-7.0) days. Table $1 \Downarrow$ shows the patients' characteristics by delirium status. Patients with delirium had significantly greater severity of disease on admission, were older, and were more likely to be male. The crude mortality was $94 / 558(17 \%)$ in patients with delirium compared with 40/554 (7\%) in those without delirium $(\mathrm{P}<0.001)$.

\section{Regression analyses}

Table $2 \Downarrow$ shows the results of the regression models. Delirium was significantly associated with mortality by logistic regression analysis (crude odds ratio $2.60,95 \%$ confidence interval 1.76 to 3.85 ; adjusted odds ratio $1.77,95 \%$ confidence interval 1.15 to 2.72 ). In time dependent, cause specific survival analysis, however, delirium had no direct effect on the daily risk of death (cause specific hazard ratio $0.64,95 \%$ confidence interval 0.39 to 1.03 ) but did result in a lower daily probability of being discharged from the intensive care unit (cause specific hazard ratio $0.53,95 \%$ confidence interval 0.46 to 0.61 ). Consequently, the risk of dying in the intensive care unit remained for longer in patients with delirium, resulting in a combined hazard of death for patients with delirium that was significantly increased (subdistribution hazard ratio $2.08,95 \%$ confidence interval 1.40 to 3.09). In contrast, once we adjusted for the evolution of disease severity before the onset of delirium in the marginal structural model analysis, delirium was no longer associated with death in the intensive care unit (subdistribution hazard ratio $1.19,95 \%$ confidence interval 0.75 to 1.89 ). Figure $3 \Downarrow$ shows the effect of delirium on the cumulative risk of death over time. By day 30, the population attributable fraction of mortality due to delirium in the intensive care unit was $7.2 \%$ (95\% confidence interval $-7.5 \%$ to $19.5 \%$ ), corresponding to an absolute case fatality of $0.9 \%$ (95\% confidence interval $-0.9 \%$ to $2.3 \%$ ).

Supplementary table 1 provides the results of the post hoc analyses using alternative definitions for delirium. The sensitivity analysis using backward imputation for patients in whom delirium assessments were obscured by the use of sedation yielded similar estimations. However, the sensitivity analysis using a delirium definition that required the derangement to persist for two or more consecutive days yielded a stronger overall association with mortality than did our primary analysis (subdistribution hazard ratio $1.67,95 \%$ confidence interval 1.13 to $2.47 ; 30$ day absolute risk difference in mortality $2.0 \%, 95 \%$ confidence interval $1.2 \%$ to $2.8 \%$ ). In this case, cause specific analysis also showed that increased mortality was mediated through a prolonged length of stay in the intensive care unit rather than by a direct effect on the daily risk of death. No signs of effect modification were found in the subgroup of 
patients who presented with sepsis, nor in relation to the severity of illness at the time of admission to the intensive care unit (see supplementary table 2).

\section{Discussion}

We estimated the mortality due to delirium in critically ill patients in intensive care while taking into account bias caused by time varying disease severity until the onset of delirium and by the competing risk of discharge. Using this approach, the population attributable mortality in the intensive care unit was estimated at $7.2 \%$ by day 30 , implying that absolute case fatality can be reduced by no more than $0.9 \%$ if we were able to completely prevent delirium in all patients.

\section{Comparison with other studies}

When we adjusted for baseline variables only our findings confirm the estimates of a twofold to threefold increased case fatality rate associated with delirium that were reported in previous studies. ${ }^{6-8} 101114-16425051$ However, as we added complexity to our analyses and adjusted for changes in disease severity before the onset of delirium, we found no association between delirium (of any duration) and mortality in the intensive care unit, which is consistent with a recent meta-analysis of clinical trials, which also showed that delirium was not associated with short term mortality ${ }^{52}$ However, results of post hoc sensitivity analyses suggest that patients who develop an episode of delirium that persists for more than two days are exposed to an overall increased risk of death in the intensive care unit. Although this finding needs further confirmation, it gives support to recent reports suggesting a fundamental distinction between rapidly reversible, sedation related delirium and severe, persistent delirium in the intensive care unit. ${ }^{53}$ Furthermore, delirium was not associated with mortality even in a prespecified subgroup of patients with sepsis. Although sepsis is often associated with delirium, it is unknown whether this so called "septic encephalopathy" is a different entity of delirium. Our results indicate that sepsis associated delirium is similar to other forms of delirium regarding mortality. In any case, however, all cause specific analyses indicated that delirium does not directly affect case fatality rates but rather that any increase in overall mortality seems to be mediated through a more protracted length of stay in the intensive care unit, exposing patients for longer to a fixed daily risk of dying (for example, due to nosocomial infections, drawbacks of prolonged sedation, or mechanical ventilation, and other "general" complications in the intensive care unit). Indeed, the average length of stay of patients without delirium was 4.0 days compared with 8.8 for patients with a short episode of delirium and 16.5 days for patients with a persistent episode. The effect of delirium on the length of stay is plausible because patients with delirium are typically less likely to interact with their environment (hampering early mobilisation), may have an increased incidence of complications (for example, self removal of catheters or tubes), and often receive drugs with sedative effects. $^{15} 505455$

\section{Strengths and limitations of this study}

Many authors have tackled the association of delirium with mortality, but only in a few studies have multivariable analyses been performed to adjust for severity of disease at baseline. ${ }^{6} 810$ To avoid immortal time bias (that is, bias due to the time varying nature of delirium), others have incorporated the time of delirium onset in their analyses. ${ }^{72}$ However, we considered it crucial to also incorporate the evolution of disease severity before the development of a delirium episode into our analyses as critically ill patients may show either rapid deterioration or reversal of organ dysfunction, both of which are likely to considerably impact on the risk of delirium onset over time. Although standard regression models can be used for this purpose, their use may result in the elimination of any potential effects of delirium on mortality if a time varying confounder is also an intermediate factor in the causal pathway that leads up to delirium, as well as to the collider-stratification bias that occurs when disease severity on a given day is influenced by the presence of delirium at a previous point in time. ${ }^{47} 56$ The marginal structural model analysis that we used is not affected by these problems.

We acknowledge some limitations of our study design. Firstly, management of delirium is largely pragmatic and may vary between centres, which may limit the generalisability of our findings. In particular, studies performed in centres using a different strategy for prevention or treatment of delirium from ours may yield disparate estimates of attributable mortality. Yet, both our case mix and the results of our initial logistic and survival regression analyses were in line with previous literature. Secondly, as is true for all observational studies, we cannot rule out the possibility that unobserved confounding might have occurred, even after accounting for a relatively large number of covariables. Thirdly, delirium can be the first symptom of an impending complication, such as sepsis acquired in the intensive care unit. ${ }^{57}{ }^{58}$ When this happens, any mortality due to this sepsis event may then be attributed falsely to delirium, since a marginal structural model analysis (deliberately) adjusts for the evolution of disease severity up until the onset of delirium. The true association of delirium with mortality might therefore be even lower than we report. Finally, by using a marginal structural model, we estimated the mortality during admission to an intensive care unit that can be expected if delirium would be prevented for the entire population. Since ordinary competing risks survival regression is a conditional rather than a marginal model, the population used in a marginal structural model differs from such unweighted analyses..$^{59}$ This dissimilarity may partly explain the observed differences in effect estimates yielded by the two approaches. However, we considered comparative reporting of these models useful to illustrate the bias that is inherently present in many commonly used analyses.

In addition to these methodological concerns, an important problem also remains about the way our study (or for that matter any study in this domain) deals with the classification of unobserved days due to coma or sedation. Because the assessment of delirium in unresponsive patients is impossible and the statistical methods that we used required a dichotomous classification of patients, we recategorised patient days on which delirium could not be observed due to coma or sedation, or both. No universally accepted methods for such reallocation exist and in fact many previous authors did not describe exactly how they dealt with these days. We chose to categorise patients who remained comatose (score of $\leq 3$ on the Richmond agitation-sedation scale) for more than 48 hours after cessation of sedation as delirious, because we believe that coma represents a state of severe "brain failure" that forms a continuum with delirium. This approach is similar to that of previous studies. ${ }^{6061}$ However, in contrast with these authors, we categorised patients who were unresponsive owing to continuous sedation (or those who were within 48 hours of stopping a sedative infusion) as non-delirious because we believed that doing otherwise would introduce bias against surgical patients who often remain sedated for at least the first hours of their stay. ${ }^{53}$ Furthermore, we 
assumed that most patients with true delirium would be detected after cessation of sedation after all, which would result in only a minor error with respect to the timing of delirium onset (rather than risking a major error of misclassifying patients as delirious when in fact they were receiving sedation for an unrelated but legitimate reason). In our sensitivity analyses we dealt with any possible misclassification error with respect to timing of delirium onset in sedated patients by using a first valid observation carried backward approach, and the results were similar.

\section{Clinical implications}

Although we did not find an association between delirium episodes of short duration and increased mortality in the intensive care unit, delirium remains an important clinical syndrome in critically ill patients and should be prevented or treated whenever possible. ${ }^{62}$ Delirium is distressing to both patients and their relatives, ${ }^{63}$ generates major costs, ${ }^{64}$ may have severe long term consequences such as cognitive impairment, ${ }^{65}$ and could cause long term mortality. ${ }^{42}$ These studies might, however, have been prone to the same bias - that is, failing to adjust for the evolution of disease severity before the onset of delirium. Most importantly, sensitivity analyses indicated that episodes of severe, persistent delirium may still be associated with an increased risk of death in the intensive care unit by day 30 , despite our overall finding of no association with delirium of any duration. The possible causal mechanisms for this association are incompletely understood, but may include autonomic dysregulation as a result of delirium causing hypotension and subsequent organ failure, immunomodulatory effects causing increased susceptibility to infections, and excessive stress responses causing increased production of corticosteroid. ${ }^{566-71}$ Nevertheless, the absolute risk increase of $2.0 \%$ after the onset of persistent delirium would translate into a number needed to treat of more than 50 , even if we were able to effectively prevent this complication by some sort of intervention. Furthermore, since this finding was based on post hoc analyses, these observations need to be confirmed in other cohorts of critically ill patients.

\section{Conclusions}

To our knowledge, this is the first study to estimate delirium associated mortality in critically ill patients using a counterfactual analysis that incorporates correction for variations in disease severity before the onset of delirium. Using this approach, the absolute attributable short term mortality associated with a delirium episode in the intensive care unit (of any duration) was much lower than previously suggested. Future studies should focus on episodes of persistent delirium and its long term sequelae rather than on acute mortality.

We thank Rolf Groenwold, Jos Frencken, and Margaret Nicholls for their excellent advice and invaluable statistical assistance.

Contributors: PMCKK, IJZ, CS, AJCS, and OLC substantially contributed to the conception and design of this study. PMCKK, IJZ, DSYO, AWvdK, MJMB, AJCS, and OLC acquired the data. PMCKK and CS had full access to all the data in the study and take responsibility for the integrity of the data and the accuracy of the data analysis. PMCKK, IJZ, CS, MJMB, AJCS, and OLC were involved in the interpretation of the data. PMCKK, IJZ, AJCS, and OLC drafted the manuscript and all authors revised it critically for important intellectual content. OLC is the guarantor for the study. All authors gave final approval of this version to be submitted.

Funding: This work was supported by the Centre for Translational Molecular Medicine (http://www.ctmm.nl), project MARS (grant 04I-201).
MB has received research funding from the Netherlands Organization of Scientific Research (NWO Vici 918.76.611). The study sponsors did not have a role in study design; in the collection, analysis, and interpretation of data; in the writing of the report; or in the decision to submit the article for publication.

Competing interests: All authors have completed the ICMJE uniform disclosure form at www.icmje.org/coi_disclosure.pdf and declare: no support from any organisation for the submitted work; no financial relationships with any organisations that might have an interest in the submitted work in the previous three years; no other relationships or activities that could appear to have influenced the submitted work.

Ethical approval: Consent was not obtained but the presented data are anonymised and risk of identification is low.

Data sharing: Patient level data, full dataset, or statistical code is available from the corresponding author at p.m.c.kleinklouwenberg@umcutrecht.nl.

Transparency: The lead author (OLC) affirms that this manuscript is an honest, accurate, and transparent account of the study being reported; that no important aspects of the study have been omitted; and that any discrepancies from the study as planned (and, if relevant, registered) have been explained.

1 Van den Boogaard M, Pickkers P, Slooter AJ, Kuiper MA, Spronk PE, van der Voort PH, et al. Development and validation of PRE-DELIRIC (PREdiction of DELIRium in ICu patients) delirium prediction model for intensive care patients: observational multicentre study. BMJ 2012;344:e420.

2 Shehabi Y, Chan L, Kadiman S, Alias A, Ismail WN, Tan MA, et al. Sedation depth and long-term mortality in mechanically ventilated critically ill adults: a prospective longitudinal multicentre cohort study. Intensive Care Med 2013;39:910-8.

3 Svenningsen H, Egerod I, Videbech P, Christensen D, Frydenberg M, Tonnesen EK. Fluctuations in sedation levels may contribute to delirium in ICU patients. Acta Anaesthesiol Scand 2013;57:288-93.

4 Zaal IJ, Spruyt CF, Peelen LM, van Eijk MM, Wientjes R, Schneider MM, et al. Intensive care unit environment may affect the course of delirium. Intensive Care Med 2013;39:481-8.

5 Riker RR, Shehabi Y, Bokesch PM, Ceraso D, Wisemandle W, Koura F, et al. Dexmedetomidine vs midazolam for sedation of critically ill patients: a randomized trial. JAMA 2009;301:489-99.

6 Ouimet S, Kavanagh BP, Gottfried SB, Skrobik Y. Incidence, risk factors and consequences of ICU delirium. Intensive Care Med 2007;33:66-73.

7 Ely EW, Shintani A, Truman B, Speroff T, Gordon SM, Harrell FE Jr, et al. Delirium as a predictor of mortality in mechanically ventilated patients in the intensive care unit. JAMA 2004:291:1753-62.

8 Lin SM, Liu CY, Wang CH, Lin HC, Huang CD, Huang PY, et al. The impact of delirium on the survival of mechanically ventilated patients. Crit Care Med 2004;32:2254-9.

9 Pisani MA, Kong SY, KasI SV, Murphy TE, Araujo KL, Van Ness PH. Days of delirium are associated with 1-year mortality in an older intensive care unit population. Am J Respir Crit Care Med 2009;180:1092-7.

10 Van den Boogaard M, Schoonhoven L, van der Hoeven JG, van Achterberg T, Pickkers $P$. Incidence and short-term consequences of delirium in critically ill patients: a prospective observational cohort study. Int J Nurs Stud 2012;49:775-83.

11 Van den Boogaard M, Peters SA, van der Hoeven JG, Dagnelie PC, Leffers P, Pickkers $\mathrm{P}$, et al. The impact of delirium on the prediction of in-hospital mortality in intensive care patients. Crit Care 2010;14:R146.

12 Lin SM, Huang CD, Liu CY, Lin HC, Wang CH, Huang PY, et al. Risk factors for the development of early-onset delirium and the subsequent clinical outcome in mechanically ventilated patients. J Crit Care 2008;23:372-9.

13 Salluh JI, Soares M, Teles JM, Ceraso D, Raimondi N, Nava VS, et al. Delirium epidemiology in critical care (DECCA): an international study. Crit Care 2010;14:R210.

14 Tomasi CD, Grandi C, Salluh J, Soares M, Giombelli VR, Cascaes S, et al. Comparison of CAM-ICU and ICDSC for the detection of delirium in critically ill patients focusing on relevant clinical outcomes. J Crit Care 2012;27:212-7.

15 Dubois MJ, Bergeron N, Dumont M, Dial S, Skrobik Y. Delirium in an intensive care unit: a study of risk factors. Intensive Care Med 2001;27:1297-304

16 Kishi $\mathrm{Y}$, Iwasaki Y, Takezawa K, Kurosawa H, Endo S. Delirium in critical care unit patients admitted through an emergency room. Gen Hosp Psychiatry 1995;17:371-9.

17 Thomason JW, Shintani A, Peterson JF, Pun BT, Jackson JC, Ely EW. Intensive care unit delirium is an independent predictor of longer hospital stay: a prospective analysis of 261 non-ventilated patients. Crit Care 2005;9:R375-81.

18 Zhang Z, Pan L, Ni H. Impact of delirium on clinical outcome in critically ill patients: a meta-analysis. Gen Hosp Psychiatry 2013;35:105-11.

19 Hernan MA, Brumback B, Robins JM. Marginal structural models to estimate the causal effect of zidovudine on the survival of HIV-positive men. Epidemiology 2000;11:561-70.

20 Bekaert M, Vansteelandt S, Mertens K. Adjusting for time-varying confounding in the subdistribution analysis of a competing risk. Lifetime Data Anal 2010;16:45-70.

21 Zaal IJ, Tekatli H, van der Kooi AW, Klijn FAM, Koek HL, van Dijk D, Slooter AJC. Classification of a daily mental status in critically ill patients for research purposes. $J$ Crit Care 2014; published online 7 Nov. doi.org/10.1016/j.jcrc.2014.10.031.

22 Sessler CN, Gosnell MS, Grap MJ, Brophy GM, O'Neal PV, Keane KA, et al. The Richmond agitation-sedation scale: validity and reliability in adult intensive care unit patients. $A m J$ Respir Crit Care Med 2002;166:1338-44.

23 Zaal IJ, Slooter AJ. Delirium in critically ill patients: epidemiology, pathophysiology, diagnosis and management. Drugs 2012;72:1457-71. 


\section{What is already known on this topic}

Numerous observational studies have suggested that delirium increases the risk of death in critically ill patients by up to threefold

\section{What this study adds}

As delirium develops in the most severely ill patients, it is crucial to adjust for evolution in disease severity until the onset of delirium Our study suggests that delirium does not cause short term mortality in critically ill patients after correction for time dependent sources of confounding

24 Van Rompaey B, Elseviers MM, Schuurmans MJ, Shortridge-Baggett LM, Truijen S, Bossaert L. Risk factors for delirium in intensive care patients: a prospective cohort study. Crit Care 2009;13:R77.

25 Pandharipande P, Shintani A, Peterson J, Pun BT, Wilkinson GR, Dittus RS, et al. Lorazepam is an independent risk factor for transitioning to delirium in intensive care unit patients. Anesthesiology 2006;104:21-6.

26 Van Eijk MM, Slooter AJ, Kesecioglu J, van der Mast RC. Delirium op de intensive care. Ned Tijdschr Geneeskd 2008;152:2768-73.

27 Agarwal V, O'Neill PJ, Cotton BA, Pun BT, Haney S, Thompson J, et al. Prevalence and risk factors for development of delirium in burn intensive care unit patients. J Burn Care Res 2010;31:706-15.

28 Pisani MA, Murphy TE, Araujo KL, Slattum P, Van Ness PH, Inouye SK. Benzodiazepine and opioid use and the duration of intensive care unit delirium in an older population. Crit Care Med 2009;37:177-83

29 Pisani MA, Murphy TE, Araujo KL, Van Ness PH. Factors associated with persistent delirium after intensive care unit admission in an older medical patient population. $J$ Crit Care 2010;25:540 e1-7.

30 Chang YL, Tsai YF, Lin PJ, Chen MC, Liu CY. Prevalence and risk factors for postoperative delirium in a cardiovascular intensive care unit. Am J Crit Care 2008;17:567-75.

31 Pandharipande P, Cotton BA, Shintani A, Thompson J, Pun BT, Morris JA Jr, et al. Prevalence and risk factors for development of delirium in surgical and trauma intensive care unit patients. J Trauma 2008;65:34-41.

32 Granberg Axell Al, Malmros CW, Bergbom IL, Lundberg DB. Intensive care unit syndrome/delirium is associated with anemia, drug therapy and duration of ventilation treatment. Acta Anaesthesiol Scand 2002;46:726-31.

33 McNicoll L, Pisani MA, Zhang Y, Ely EW, Siegel MD, Inouye SK. Delirium in the intensive care unit: occurrence and clinical course in older patients. J Am Geriatr Soc 2003;51:591-8.

34 Seaman JS, Schillerstrom J, Carroll D, Brown TM. Impaired oxidative metabolism precipitates delirium: a study of $101 \mathrm{ICU}$ patients. Psychosomatics 2006;47:56-61.

35 Woods JC, Mion LC, Connor JT, Viray F, Jahan L, Huber C, et al. Severe agitation among ventilated medical intensive care unit patients: frequency, characteristics and outcomes. Intensive Care Med 2004;30:1066-72.

36 Aldemir M, Ozen S, Kara IH, Sir A, Bac B. Predisposing factors for delirium in the surgical intensive care unit. Crit Care 2001;5:265-70.

37 Pisani MA, Murphy TE, Van Ness PH, Araujo KL, Inouye SK. Characteristics associated with delirium in older patients in a medical intensive care unit. Arch Intern Med 2007;167:1629-34.

38 Jaber S, Chanques G, Altairac C, Sebbane M, Vergne C, Perrigault PF, et al. A prospective study of agitation in a medical-surgical ICU: incidence, risk factors, and outcomes. Chest 2005;128:2749-57

39 Zimmerman JE, Kramer AA, McNair DS, Malila FM. Acute Physiology and Chronic Health Evaluation (APACHE) IV: hospital mortality assessment for today's critically ill patients. Crit Care Med 2006:34:1297-310.

40 Klein Klouwenberg PM, Ong DS, Bos LD, de Beer FM, van Hooijdonk RT, Huson MA, et al. Interobserver agreement of Centers for Disease Control and Prevention criteria for classifying infections in critically ill patients. Crit Care Med 2013;41:2373-8.

41 Engels JM, Diehr P. Imputation of missing longitudinal data: a comparison of methods. $J$ Clin Epidemiol 2003:56:968-76.

42 Shintani AK, Girard TD, Eden SK, Arbogast PG, Moons KGM, Ely EW. Immortal time bias in critical care research: application of time-varying Cox regression for observational cohort studies. Crit Care Med 2009;37:2939-45.

43 Wolkewitz M, Vonberg R, Grundmann H, Beyersmann J, Gastmeier P, Bärwolff S, et al. Risk factors for the development of nosocomial pneumonia and mortality on intensive care units: application of competing risks models. Crit Care 2008;12:R44.

44 Wolkewitz M, Cooper BS, Bonten MJ, Barnett AG, Schumacher M. Interpreting and comparing risks in the presence of competing events. BMJ 2014;349:g5060.

45 Fine JP, Gray RJ. A proportional hazards model for the subdistribution of a competing risk. J Am Stat Assoc 1999;94:496-509.

46 Robins JM, Hernan MA, Brumback B. Marginal structural models and causal inference in epidemiology. Epidemiology 2000;11:550-60.

47 Bekaert M, Timsit JF, Vansteelandt S, Depuydt P, Vesin A, Garrouste-Orgeas M, et al. Attributable mortality of ventilator-associated pneumonia: a reappraisal using causal analysis. Am J Respir Crit Care Med 2011;184:1133-9.

48 Van der Wal WM, Geskus RB. IPW: an R package for inverse probability weighting. $J$ Stat Software 2011;43:1-23.

49 Freedman DA. On the so-called "huber sandwich estimator" and "robust standard errors". Am Stat 2006;60:299-302
50 Balas MC, Deutschman CS, Sullivan-Marx EM, Strumpf NE, Alston RP, Richmond TS. Delirium in older patients in surgical intensive care units. J Nurs Scholarsh 2007;39:147-54 51 Shi CM, Wang DX, Chen KS, Gu XE. Incidence and risk factors of delirium in critically ill patients after non-cardiac surgery. Chin Med J (Engl) 2010;123:993-9.

52 Al-Qadheeb NS, Balk EM, Fraser GL, Skrobik Y, Riker RR, Kress JP, et al. Randomized ICU trials do not demonstrate an association between interventions that reduce delirium duration and short-term mortality: a systematic review and meta-analysis. Crit Care Med 2014;42:1442-54

53 Patel SB, Poston JT, Pohlman A, Hall JB, Kress JP. Rapidly reversible, sedation-related delirium versus persistent delirium in the intensive care unit. Am J Respir Crit Care Med 2014;189:658-65.

54 Girard TD, Kress JP, Fuchs BD, Thomason JW, Schweickert WD, Pun BT, et al. Efficacy and safety of a paired sedation and ventilator weaning protocol for mechanically ventilated patients in intensive care (Awakening and Breathing Controlled trial): a randomised controlled trial. Lancet 2008;371:126-34.

55 Schweickert WD, Pohlman MC, Pohlman AS, Nigos C, Pawlik AJ, Esbrook CL, et al. Early physical and occupational therapy in mechanically ventilated, critically ill patients: a randomised controlled trial. Lancet 2009;373:1874-82.

56 Greenland S. Quantifying biases in causal models: classical confounding vs collider-stratification bias. Epidemiology 2003;14:300-6.

57 Gofton TE, Young GB. Sepsis-associated encephalopathy. Nat Rev Neurol 2012;8:557-66.

58 Martin BJ, Buth KJ, Arora RC, Baskett RJ. Delirium as a predictor of sepsis in post-coronary artery bypass grafting patients: a retrospective cohort study. Crit Care 2010;14:R171.

59 Kurth T, Walker AM, Glynn RJ, Chan KA, Gaziano JM, Berger K, et al. Results of multivariable logistic regression, propensity matching, propensity adjustment, and propensity-based weighting under conditions of nonuniform effect. Am J Epidemiol 2006;163:262-70.

60 Hughes CG, Pandharipande PP. Review articles: the effects of perioperative and intensive care unit sedation on brain organ dysfunction. Anesth Analg 2011;112:1212-7.

61 Pandharipande PP, Pun BT, Herr DL, Maze M, Girard TD, Miller RR, et al. Effect of sedation with dexmedetomidine vs lorazepam on acute brain dysfunction in mechanically ventilated patients: the MENDS randomized controlled trial. JAMA 2007;298:2644-53.

62 Ely EW. The Modifying the Impact of ICU-Associated Neurological Dysfunction-USA (MIND-USA) Study: ClinicalTrials.gov. National Library of Medicine, 2013:NCT01211522.

63 Breitbart W, Gibson C, Tremblay A. The delirium experience: delirium recall and delirium-related distress in hospitalized patients with cancer, their spouses/caregivers, and their nurses. Psychosomatics 2002;43:183-94.

64 Strijbos MJ, Steunenberg B, van der Mast RC, Inouye SK, Schuurmans MJ. Design and methods of the Hospital Elder Life Program (HELP), a multicomponent targeted intervention to prevent delirium in hospitalized older patients: efficacy and cost-effectiveness in Dutch health care. BMC Geriatr 2013;13:78.

65 Pandharipande PP, Girard TD, Jackson JC, Morandi A, Thompson JL, Pun BT, et al Long-term cognitive impairment after critical illness. N Engl J Med 2013;369:1306-16.

66 Trzepacz PT. Update on the neuropathogenesis of delirium. Dement Geriatr Cogn Disord 1999;10:330-4.

67 Trzepacz PT. Is there a final common neural pathway in delirium? Focus on acetylcholine and dopamine. Semin Clin Neuropsychiatry 2000;5:132-48.

68 Hshieh TT, Fong TG, Marcantonio ER, Inouye SK. Cholinergic deficiency hypothesis in delirium: a synthesis of current evidence. J Gerontol A Biol Sci Med Sci 2008:63:764-72.

69 Steiner LA. Postoperative delirium. Part 1: pathophysiology and risk factors. Eur J Anaesthesiol 2011;28:628-36.

70 Van Gool WA, van de Beek D, Eikelenboom P. Systemic infection and delirium: when cytokines and acetylcholine collide. Lancet 2010;375:773-5.

71 Maclullich AM, Ferguson KJ, Miller T, de Rooij SE, Cunningham C. Unravelling the pathophysiology of delirium: a focus on the role of aberrant stress responses. J Psychosom Res 2008:65:229-38.

\section{Accepted: 30 October 2014}

\section{Cite this as: BMJ 2014;349:g6652}

This is an Open Access article distributed in accordance with the Creative Commons Attribution Non Commercial (CC BY-NC 4.0) license, which permits others to distribute, remix, adapt, build upon this work non-commercially, and license their derivative works on different terms, provided the original work is properly cited and the use is non-commercial. See: http://creativecommons.org/licenses/by-nc/4.0/. 


\section{Tables}

Table 1| Patient characteristics by delirium status. Values are numbers (percentages) unless stated otherwise

\begin{tabular}{|c|c|c|c|}
\hline Characteristics & Never delirium $(n=554)$ & Ever delirium $(n=558)$ & $P$ value \\
\hline Median (IQR) age (years) & $61(49-69)$ & $64(54-74)$ & $<0.001$ \\
\hline Men & $316(57)$ & $356(64)$ & 0.02 \\
\hline White ethnicity & $535(97)$ & $534(96)$ & 0.29 \\
\hline Current alcohol misuse* & $12(2)$ & $33(6)$ & $<0.001$ \\
\hline Median (IQR) Charlson comorbidity index & $5.4(0.0-10.2)$ & $7.1(1.0-11.4)$ & $<0.001$ \\
\hline Previous ICU admission† & $83(15)$ & $91(16)$ & 0.54 \\
\hline \multicolumn{4}{|l|}{ Admission type: } \\
\hline Medical & $253(46)$ & $266(48)$ & \multirow[t]{3}{*}{0.26} \\
\hline Scheduled surgery & $165(30)$ & $142(25)$ & \\
\hline Emergency surgery & $136(24)$ & $150(27)$ & \\
\hline \multicolumn{4}{|l|}{ Medical specialty: } \\
\hline General surgery & $213(38)$ & $211(38)$ & \multirow[t]{4}{*}{$<0.001$} \\
\hline Cardiology and cardiothoracic surgery & $165(30)$ & $170(30)$ & \\
\hline Internal medicine & $105(19)$ & $131(23)$ & \\
\hline Other & $71(13)$ & $46(9)$ & \\
\hline Sepsis at admission & $190(34)$ & $306(55)$ & $<0.001$ \\
\hline Mechanical ventilation at admission & $470(85)$ & $510(91)$ & $<0.001$ \\
\hline Median (IQR) APACHE IV score & $63(48-81)$ & $79(62-97)$ & $<0.001$ \\
\hline Median (IQR) length of stay (days) & $3(2-5)$ & $9(5-18)$ & $<0.001$ \\
\hline ICU case fatality & $40(7)$ & $94(17)$ & $<0.001$ \\
\hline
\end{tabular}

$\mathrm{IQR}=$ interquartile range; ICU=intensive care unit; APACHE=acute physiology and chronic health evaluation.

*Defined as alcohol consumption $>40 \mathrm{~g}$ alcohol daily.

†Defined as previous admission to the intensive care unit during current hospital stay. 
Table 2| Effect estimates for association between delirium and mortality in intensive care unit using various statistical approaches

Variables Logistic regression Competing risks survival regression Marginal structural model

Adjustment factors:

\begin{tabular}{lllll}
\hline Baseline covariables & Yes & Yes & Yes \\
\hline Time varying onset of delirium & No & Yes & Yes \\
\hline Competing risks of death and discharge & No & Yes & Yes \\
\hline Evolution of disease before delirium & No & No & Yes
\end{tabular}

onset $^{*}$

Effect estimate†:

\begin{tabular}{llll}
\hline Crude & $2.60(1.76$ to 3.85$)$ & $3.14(2.32$ to 5.04$)$ & $3.14(2.32$ to 5.04$) \S$ I \\
\hline Adjusted $^{\star *}$ & $1.77(1.15$ to 2.72$)$ & $2.08(1.40$ to 3.09$)$ & $1.19(0.75$ to 1.89$) \dagger † \ddagger \ddagger$ \\
\hline
\end{tabular}

*Logistic regression and survival analysis can also be used to correct for evolution of disease severity; however, over-adjustment and collider stratification bias might occur. The marginal structural model prevents these biases. ${ }^{19}$

†Logistic regression analysis gives an odds ratio, whereas survival analysis and marginal structural model provide a subdistribution hazard ratio. ‡Delirium was included as a time dependent variable in competing risks survival regression and marginal structural models.

§Crude subdistribution hazard ratio of marginal structural model was calculated assuming the weights to be equal to 1 and is therefore equal to estimation of competing risks survival analysis.

ๆAdjusted cause specific hazard ratios of competing risks survival regression were 0.64 ( $95 \%$ confidence interval 0.39 to 1.03 ) for mortality and 0.53 ( 0.46 to 0.61 ) for discharge.

** Multivariable analysis was adjusted for baseline variables (age, sex, Charlson comorbidity index, acute physiology and chronic health evaluation IV score, admission type, and sepsis on admission). The marginal structural model was furthermore adjusted to time varying variables: sequential organ failure assessment score, sepsis status, temperature, sodium, urea concentration, acidosis, haematocrit, mechanical ventilation, and sedative and analgesic drugs. ††Adjusted cause specific hazard ratios of the marginal structural model analysis were 0.38 (95\% confidence interval 0.22 to 0.65 ) for mortality and 0.65 ( 0.55 to 0.76) for discharge.

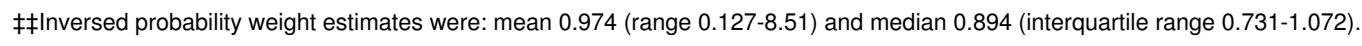




\section{Figures}

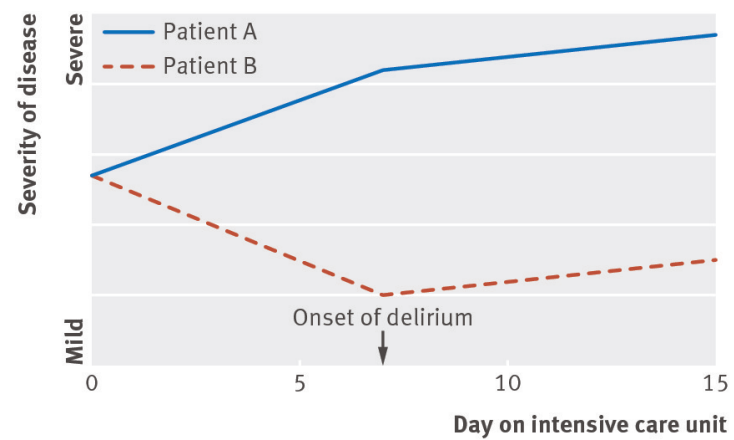

Fig 1 Evolution of disease severity before onset of delirium in two hypothetical patients admitted to the intensive care unit. Both patients have similar severity of disease, but the condition of patient A worsens, whereas that of patient B improves. As delirium preferentially develops in more severely ill patients, confounding occurs when disease severity after baseline is not adjusted for in the analysis. Logistic regression and survival analysis adjusts for baseline variables at $t=0$ only. A marginal structural model adjusts for changes in disease severity until the onset of delirium (area to left of arrow), but not thereafter (area to right of arrow)

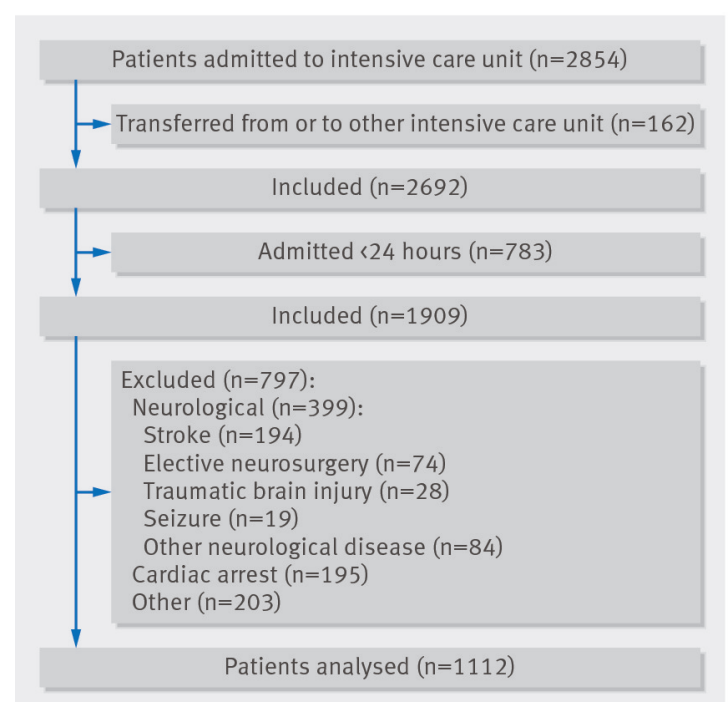

Fig 2 Flowchart of patient inclusion. "Other neurological disease" includes patients with encephalitis, encephalopathy, coma, or hydrocephalus. "Other" includes patients with premorbid neurological conditions or patients in whom delirium assessments could not be made owing to, for example, language barriers or severe mental retardation 
- Observed mortality

- - - Estimated mortality without delirium

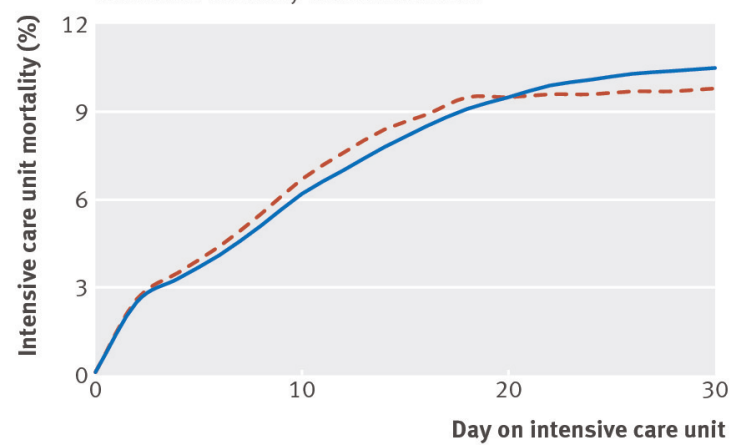

Fig 3 Cumulative incidence of observed and estimated mortality in the intensive care unit This figure represents the expected mortality in the whole cohort estimated by the cumulative incidence function in the absence and presence of delirium. A competing risks analysis was used to adjust for informative censoring, and a marginal structural method for evolution of disease 